\title{
Synchrotron SAXS Studies on Morphology Formation in a Binary Blend of Poly( $\varepsilon$-caprolactone) Homopolymer and Poly( $\varepsilon$-caprolactone)-block-Polybutadiene Copolymer
}

\author{
Michiaki AKABA and Shuichi NOJIMA ${ }^{\dagger}$ \\ Department of Polymer Chemistry, Tokyo Institute of Technology, \\ H-125, 2-12-1 Ookayama, Meguro-ku, Tokyo 152-8552, Japan
}

(Received December 1, 2004; Accepted April 14, 2005; Published July 15, 2005)

\begin{abstract}
The process of morphology formation in a binary blend of poly( $\varepsilon$-caprolactone) homopolymer (PCL) and $\operatorname{poly}(\varepsilon$-caprolactone)-block-polybutadiene copolymer (PCL- $b$-PB) has been investigated by synchrotron smallangle X-ray scattering (SR-SAXS). This blend shows an UCST-type phase separation and the crystallization of PCL chains (i.e., PCL and PCL blocks in PCL- $b$-PB) at a same temperature range, so that these two factors may work simultaneously to yield a complicated morphology formation. When the weight fraction of PCL $\left(\phi_{\mathrm{PCL}}\right)$ is small $\left(\phi_{\mathrm{PCL}}<0.2\right)$ or large $\left(\phi_{\mathrm{PCL}}>0.8\right)$, the blend can directly be quenched into crystallization temperatures without passing through the UCST region. Time-resolved SAXS curves in this case show that overall morphology formation is driven by the crystallization of PCL chains, where a crystallized PCL region always coexists with a crystallized PCL- $b$-PB region and the volume ratio of two regions is constant throughout. [DOI 10.1295/polymj.37.464]

KEY WORDS Morphology Formation / Crystallization / Phase Separation / Crystallineamorphous Diblock Copolymer / Small-Angle X-Ray Scattering / Synchrotron Radiation /
\end{abstract}

Morphology formation in polymer blends is controlled by many factors such as liquid-liquid phase separation between components, crystallization of constituent polymers, and microphase separation of block copolymers. ${ }^{1,2}$ This morphology formation has widely been investigated when only one factor works to yield a final morphology in the blend. When two or more factors work simultaneously in the morphology formation, however, the mechanism becomes extremely complicated. If we consider a binary blend consisting of amorphous block copolymers and homopolymers, for example, liquid-liquid phase separation and microphase separation may act simultaneously, where the phase diagram is also complicated depending on the combination of macro- (or liquid-liquid) and micro-phase separation regions. ${ }^{3,4}$ Some experimental results have been reported so far to clarify the mechanism of such morphology formation. ${ }^{3-8}$

When we suppose a binary blend consisting of crystalline and amorphous homopolymers, the morphology formation is driven by a cooperative effect of liquid-liquid phase separation and crystallization, and eventually we have another kind of complicated morphology formation. That is, the domain morphology caused by the phase separation has to coexist with the lamellar morphology (i.e., an alternating structure composed of thin lamellar crystals and amorphous layers) and also spherulite structure owing to the crystallization of constituent homopolymers. There are some experimental studies on the bahavior of such morphology formation ${ }^{9-11}$ and resulting morphology ${ }^{12-16}$ in binary blends of crystalline and amorphous homopolymers.

We have previously investigated the process of morphology formation in a binary blend of $\operatorname{poly}(\varepsilon-$ caprolactone) homopolymer (PCL) and polystyrene oligomer (PSO) by using synchrotron small-angle Xray scattering (SR-SAXS), ${ }^{9}$ where this blend had an UCST-type phase separation and the crystallization of PCL at a same temperature range. We found that when the homogeneous blend was quenched into crystallization temperatures without passing through the UCST region, total morphology formation was driven by the crystallization of PCL and the system consisted of two regions at all times, that is, amorphous region mainly made of PSO and crystallized region consisting of PCL lamellae and amorphous layers, as schematically illustrated in Figure 1a. These two regions increased in volume fraction with increasing crystallization time $(t)$ but the volume ratio of two regions in the blend was constant irrespective of $t$ (Figures $1 \mathrm{~b}$ and $1 \mathrm{c})$. We could get information on this morphology formation from the crystallized region, since it had a definite structure (i.e., lamellar morphology) inside and accordingly we obtained the SAXS peak. But the amorphous region did not give any SAXS peak, so that we could not get information on the morphology formation from this amorphous region.

In this study we employ a binary blend of PCL and poly( $\varepsilon$-caprolactone)-block-polybutadiene copolymer

${ }^{\dagger}$ To whom correspondence should be addressed (TEL: +81-3-5734-2132, Fax: +81-3-5734-2888, E-mail: snojima@polymer.titech.ac.jp). 


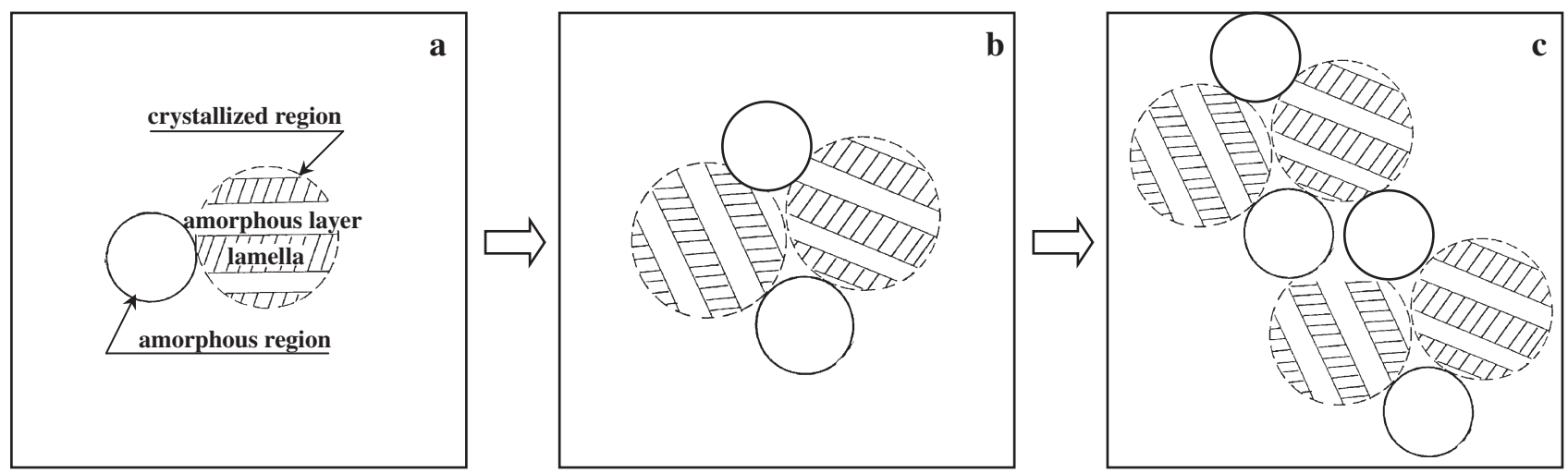

Figure 1. Schematic drawing for the morphology formation by a combined effect of crystallization and phase separation in a binary blend of crystalline and amorphous homopolymers. The matrix consists of homogeneous blends.

(PCL- $b$-PB), that is, we use PCL- $b$-PB instead of PSO, because PCL- $b$-PB yields characteristic structures before and after the crystallization of PCL blocks and therefore we can get information from this region as well as the PCL region on the morphology formation, which is again pursued by SR-SAXS as a function of $t$ at various compositions and temperatures. As a result, we study the morphology formation in a binary blend of two crystalline polymers with an UCST-type phase separation between components. Though there is a limited number of studies on the crystallization behavior and resulting morphology for completely miscible binary blends, ${ }^{17-20}$ it is a new point to investigate the process of morphology formation by the combined effect of crystallization and phase separation by using two crystalline polymers.

\section{EXPERIMENTAL}

\section{Samples}

PCL was purchased from Polymer Source Co. and PCL- $b$-PB was synthesized by a successive anionic polymerization under vacuum. Details of PCL- $b$-PB synthesis are described elsewhere. ${ }^{21,22}$ The molecular characteristics of PCL and PCL- $b$-PB are shown in Table I.

The blend was prepared by a solution-casting method using benzene as a common solvent. First an appropriate amount of PCL and PCL- $b$-PB was dis-

Table I. Molecular characteristics of the samples used in this study

\begin{tabular}{lrcccc}
\hline Sample & Total $M_{\mathrm{n}}{ }^{\mathrm{a}}$ & $M_{\mathrm{w}} / M_{\mathrm{n}}{ }^{\mathrm{b}}$ & $\begin{array}{c}\text { PCL:PB } \\
(\text { vol. \%) }\end{array}$ & $\begin{array}{c}\text { Ethyl Branch } \\
(\mathrm{mol} \%)\end{array}$ & $\begin{array}{c}T_{\mathrm{m}}{ }^{\mathrm{d}} \\
\left({ }^{\circ} \mathrm{C}\right)\end{array}$ \\
\hline PCL- $b$-PB & 13,000 & 1.16 & $66: 34$ & 7 & 54 \\
PCL & 3,500 & 1.50 & - & - & 58 \\
\hline
\end{tabular}

${ }^{a}$ Determined by membrane osmometry. ${ }^{b}$ Determined by GPC. ${ }^{\mathrm{c}}$ Determined by ${ }^{1} \mathrm{H}$ NMR. ${ }^{\mathrm{d}}$ Determined by DSC. solved in benzene, cast on a glass plate, and then benzene was evaporated in atmosphere at room temperature for $3 \mathrm{~d}$. The blend was further dried under vacuum for $24 \mathrm{~h}$ in order to completely remove the solvent.

\section{Cloud Point and Melting Point Measurements}

The cloud point was determined by the temperature at which the transmitted intensity from an $\mathrm{He}-\mathrm{Ne}$ laser (Japan Science Engineering Co.) started to decrease when the sample temperature was decreased at a constant rate of $1{ }^{\circ} \mathrm{C} / \mathrm{min}$ from a microphaseseparated melt $\left(>120^{\circ} \mathrm{C}\right)$.

The melting temperature of PCL chains (i.e., PCL and PCL blocks in PCL- $b$-PB) was obtained by differential scanning calorimetry (DSC, PerkinElmer Pyris I). The blend was first annealed at $120^{\circ} \mathrm{C}$ for $15 \mathrm{~min}$ to erase the morphology owing to the crystallization of PCL chains, quenched into $40^{\circ} \mathrm{C}$ at a maximum cooling rate of $500^{\circ} \mathrm{C} / \mathrm{min}$, annealed (or crystallized) at $40^{\circ} \mathrm{C}$ for a long time, and finally heated at a rate of $10^{\circ} \mathrm{C} / \mathrm{min}$ up to $120^{\circ} \mathrm{C}$. The melting temperature was evaluated from the maximum of endothermic peak.

We constructed a quasi phase diagram of this blend by the combination of cloud points and melting points (Figure 2) to get information on the morphology formation for SR-SAXS measurements.

\section{SR-SAXS Measurements}

The SR-SAXS experiment was carried out at Photon Factory in High-Energy Accelerator Research Organization, Tsukuba Japan, with a small-angle X-ray equipment for solution (SAXES) installed at beam line BL-10C. Details of the optics and the instrumentation were described elsewhere. ${ }^{9,21}$ The incident beam intensity with wave length $\lambda=0.1488 \mathrm{~nm}$ was monitored before and after the sample by two ionization chambers for the evaluation of frame-by-frame transmission factors of the blend and also for the cor- 
rection of a minor decrease in intensity during measurements. The detector was a one-dimensional position sensitive proportional counter (PSPC) and data accumulation time was $10 \mathrm{~s}$ to get each SAXS curve during the morphology formation. The background scattering and the Lorentz factor were taken into account, and finally the relative intensity was obtained as a function of wave number $s\left(=\frac{2}{\lambda} \sin \theta, 2 \theta\right.$ : scattering angle).

\section{Analysis of SR-SAXS Data}

The SR-SAXS curves during the morphology formation are extremely complicated because they consist of three scattering peaks and also an increasing intensity toward $s \rightarrow 0$. These scattering curves can be fully understood from Figure 1; (a) the crystallized PCL region consisting of PCL lamellae and amorphous layers yields the SAXS peak at higher angle (indicated by $\mathrm{C}$ in Figure 3), (b) the amorphous region in Figure 1 is replaced with a crystallized PCL- $b$-PB region in this blend and therefore it makes the lamellar morphology to give the SAXS peak at lower angle (B in Figure 3), and (c) the remaining (or still not crystallized) microdomain structure in the matrix also gives the SAXS peak at the angle between two former peaks (A in Figure 3), which reduces in intensity and eventually disappears after crystallization. In addition, a heterogeneous structure, that is, a two-phase structure consisting of two crystallized regions (PCL and PCL- $b$-PB regions), gives a monotonically increasing scattering toward $s \rightarrow 0$ (D in Figure 3).

The scattering from the two-phase structure $\left(I_{b}(s)\right)$ is successfully approximated by the Debye-Bueche equation, ${ }^{9,16}$

$$
I_{b}(s) \propto \frac{V(t)\left\langle\eta^{2}\right\rangle \xi^{3}}{\left(1+4 \pi^{2} s^{2} \xi^{2}\right)^{2}}
$$

and

$$
V(t)=V_{\mathrm{PCL}}(t) V_{\mathrm{PCL}-b-\mathrm{PB}}(t) /\left\{V_{\mathrm{PCL}}(t)+V_{\mathrm{PCL}-b-\mathrm{PB}}(t)\right\}(2)
$$

where $\left\langle\eta^{2}\right\rangle$ is the mean square of electron density difference between two regions, $\xi$ is a correlation length defining the size of heterogeneity in the two-phase structure, and $V_{\mathrm{PCL}}(t)$ and $V_{\mathrm{PCL}-b-\mathrm{PB}}(t)$ are the volume of the crystallized PCL and PCL- $b$-PB regions at $t$, respectively.

First we subtracted the scattering from the twophase structure by using eq 1 and then two scattering peaks arising from the PCL and PCL- $b$-PB regions were approximated by the Lorentz function,

$$
I(s)=\frac{2 A}{\pi W}\left\{1+\frac{4}{W^{2}}\left(s-s_{\max }\right)^{2}\right\}^{-1}
$$

where $A$ is the peak area, $W$ is the full width at half maximum (FWHM) of the peak intensity, and $s_{\max }$ is the wave number at peak position. Equation 3 is usually used to approximate diffraction intensities from crystalline materials in order to empirically characterize each peak ${ }^{23}$ and therefore it does not have any theoretical basis.

The approximation of SAXS peaks with eq 3 was rather difficult because the peak from crystallized regions was small at the beginning of crystallization. In particular, the SAXS peak arising from the crystallized PCL- $b$-PB region was partially overlapped with that from the microdomain structure, which leads to a moderate error in the evaluated maximum intensity. (Figures 4, 5 and 7). However, we had information on the angular position and intensity of the final SAXS peak arising from the crystallized PCL- $b$-PB region, so that the $t$ dependence of peak intensity thus obtained is adequately reliable.

\section{RESULTS AND DISCUSSION}

\section{Cloud Points and Melting Points}

Figure 2 shows the phase diagram of this blend composed of cloud points owing to the phase separation between PCL and PCL- $b$-PB and melting points of PCL chains. Melting temperatures of PCL chains are about $60^{\circ} \mathrm{C}$ irrespective of composition and they meet with the cloud point curve at $\phi_{\mathrm{PCL}}=0.2$ and 0.8 , where $\phi_{\mathrm{PCL}}$ is the weight fraction of PCL in the blend. This means that blends with $\phi_{\mathrm{PCL}} \leq 0.2$ or $\phi_{\mathrm{PCL}} \geq 0.8$ can directly be quenched into crystallization temperatures without passing through the UCST region, where the phase separation between PCL and

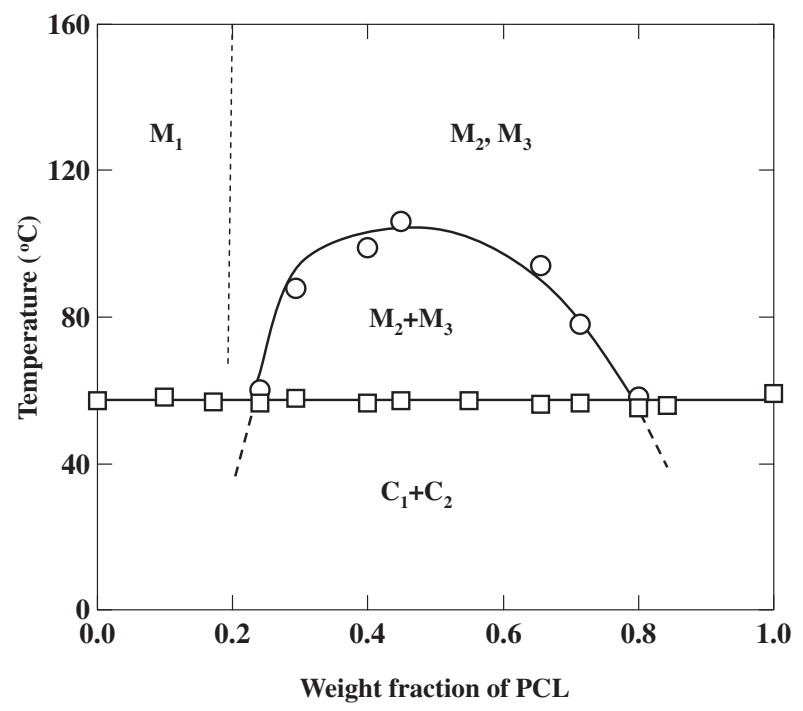

Figure 2. Cloud points $(\bigcirc)$ and melting points $(\square)$ of PCL chains (i.e., PCL block + PCL homopolymer) plotted against the weight fraction of PCL. $\mathrm{M}_{1}, \mathrm{M}_{2}$, and $\mathrm{M}_{3}$ represent lamellar, cylindrical, and spherical microdomain structures, respectively, and $\mathrm{C}_{1}$ and $\mathrm{C}_{2}$ stand for the crystallized PCL- $b$-PB and PCL phases. 
PCL- $b$-PB occurs before crystallization. Therefore, we can exclude the effect of pre-existing phase-separated structures on the crystallization of PCL chains by using blends with $\phi_{\mathrm{PCL}} \leq 0.2$ or $\phi_{\mathrm{PCL}} \geq 0.8$, though the existence of this UCST region might affect the morphology formation even for these blends.

The region above the UCST phase separation takes various microdomain structures depending on $\phi_{\mathrm{PCL}}$, as depicted by $\mathrm{M}_{1}, \mathrm{M}_{2}$, and $\mathrm{M}_{3}$ in Figure 2, which represent lamellar, cylindrical, and spherical phases, respectively. They were evaluated from the angular positions of diffraction peaks obtained at $120^{\circ} \mathrm{C}$, though the boundary between $\mathrm{M}_{2}$ and $\mathrm{M}_{3}$ was unclear because of the lack of higher-order diffractions from microdomain structures. We have to consider combined effects among microphase separation, USCTtype phase separation, and crystallization when we construct a perfect phase diagram of this blend, which will be extremely complicated judging from theoretical phase diagrams proposed for a binary blend of amorphous homopolymers and diblock copolymers. ${ }^{3,4}$ In our blend, however, the microdomain structure does not affect the following morphology formation at all, that is, it does not confine the subsequent crystallization within it, though this phenomenon is usually observed in high-molecular weight crystallineamorphous diblock copolymers. ${ }^{24,25}$ Therefore the phase diagram shown in Figure 2 is enough for this study to qualitatively understand the morphology formation in our blend.

\section{SR-SAXS Curves}

Figure 3 shows typical time-resolved SAXS curves during morphology formation when the blend with $\phi_{\mathrm{PCL}}=0.8$ is quenched from $120^{\circ} \mathrm{C}$ into $40^{\circ} \mathrm{C}$. Immediately after quenching, we can observe the SAXS peak (denoted by A), which arises from the mi-

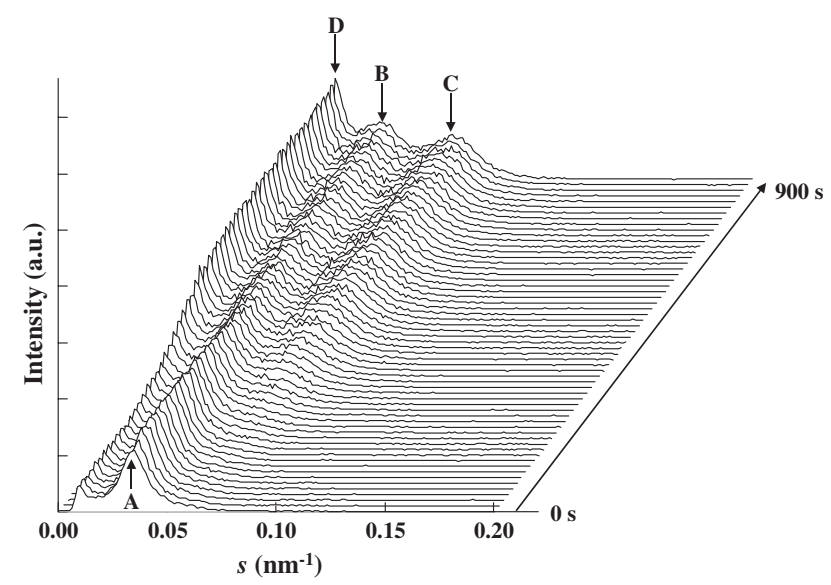

Figure 3. Time-resolved SAXS curves during crystallization for the blend with $\phi_{\mathrm{PCL}}=0.8$ after quenching from the microphase-separated melt into $T_{\mathrm{c}}=40^{\circ} \mathrm{C}$. crodomain structure existing at $120^{\circ} \mathrm{C}$. This peak decreases in intensity with $t$, and simultaneously two peaks at higher (C) and lower (B) angles appear, which come from the crystallized PCL and PCL- $b$ $\mathrm{PB}$ regions, respectively, judging from the fact that the angular position of these two peaks almost corresponds to that of crystallized neat PCL and PCL- $b$-PB (see Figure $4 a$ ). In the blend with $\phi_{\mathrm{PCL}}=0.1$ and 0.2 , the SAXS peak from the microdomain structure is sharp and strong, and we can not observe the higher angle peak originating from the crystallized PCL region, for which two factors, (1) a small fraction of the crystallized PCL region in the blend and (2) a small difference in electron density between PCL crystals and amorphous PCL layers compared with the crystallized PCL- $b$-PB region, are responsible.

In addition, we can observe a monotonically increasing scattering toward $s \rightarrow 0$ in Figure 3, which arises from the mosaic structure consisting of the crystallized PCL- $b$-PB and PCL regions, as demonstrated in our previous studies. ${ }^{9,21}$ The size of heterogeneity for this mosaic structure will be evaluated later as a function of $t$ to discuss the morphological development in this system.

The $t$ dependence of spacing and normalized inten-
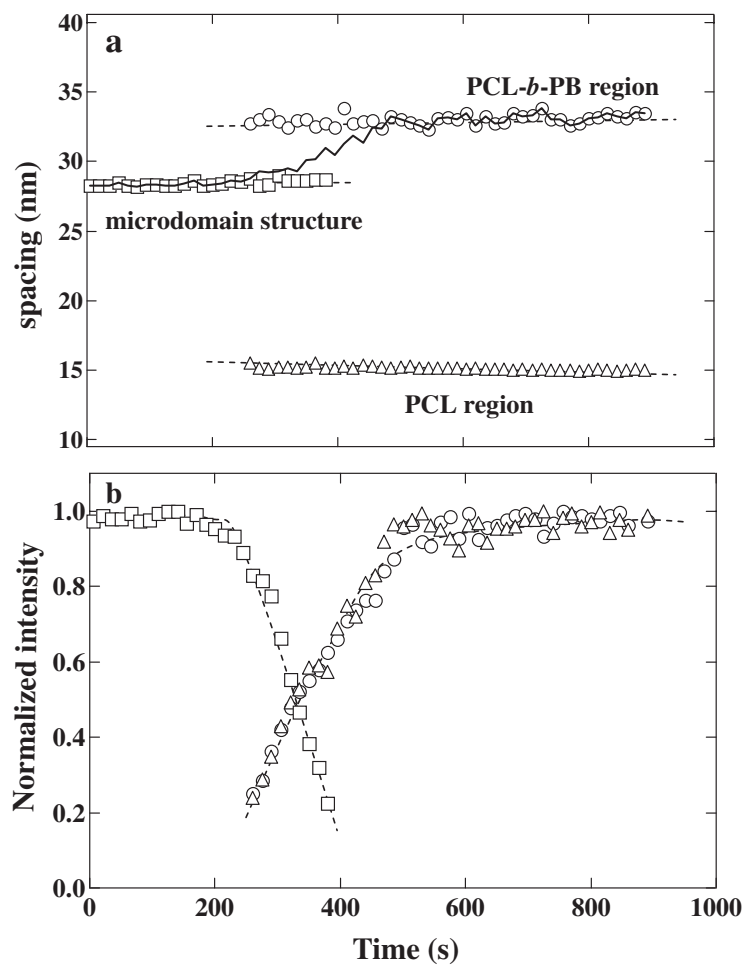

Figure 4. Spacings (a) and normalized intensities (b) for the microdomain structure $(\square)$, crystallized PCL- $b$-PB region $(\bigcirc)$, and crystallized PCL region $(\triangle)$ plotted against $t$ for the blend with $\phi_{\mathrm{PCL}}=0.8$ crystallized at $40^{\circ} \mathrm{C}$. The solid curve in panel (a) represents the spacing when the SAXS peak is approximated by a single Lorentz function (eq 3 ). 
sity, the peak intensity divided by the final intensity, for each SAXS peak is plotted in Figure 4 for the blend with $\phi_{\mathrm{PCL}}=0.8$. The angular position of SAXS peaks from the crystallized PCL- $b$-PB region is extremely close to that from the microdomain structure, so that peak separation is difficult when two peaks coexist in the middle stage of morphology formation $(250<t$ $<400$ s). An apparent change of spacing is shown by a solid curve in Figure 4a, where the SAXS peak is approximated by a single Lorentz function.

It is important to point out that the crystallization in the PCL and PCL- $b$-PB regions proceeds in a same fashion because normalized peak intensities make one master curve (Figure 4b), though the crystallization rate of neat PCL is significantly faster than that of neat PCL- $b$-PB. This is a new finding in this study, and indicates that two regions appear by a simultaneous crystallization of PCL chains. The UCST-type phase-separated regions above $T m$ indirectly affects this morphology formation at $40^{\circ} \mathrm{C}$, because we can not observe such simultaneous crystallization for completely miscible crystalline/crystalline homopolymer blends. ${ }^{17-20}$

\section{Overall Rate of Morphology Formation}

It is concluded in our previous study ${ }^{9}$ on a binary blend of PCL and PSO that total morphology formation is driven by the crystallization of PCL and the phase separation between PCL and PSO affects the details of resulting morphology. This conclusion comes from the fact that the normalized peak intensity for various blends plotted against reduced time (i.e., crystallization time divided by half-time of crystallization) is identical with that for PCL.

We check here whether the crystallization of PCL chains drives the morphology formation or not in our blend. Figure 5 shows the normalized peak intensity arising from the PCL- $b$-PB region plotted against $t$ at $40{ }^{\circ} \mathrm{C}$ for various blends indicated. Note that we

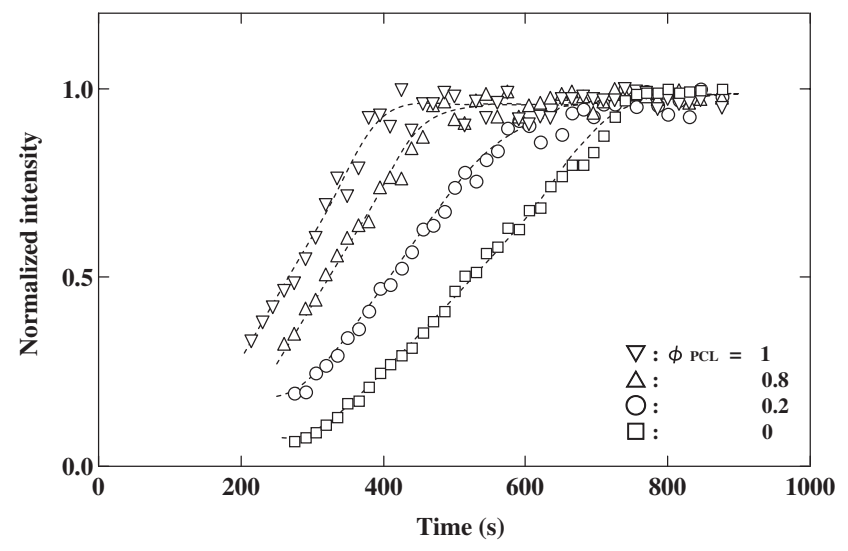

Figure 5. Normalized intensity arising from the crystallized PCL- $b$-PB region plotted against $t$ for various blends.

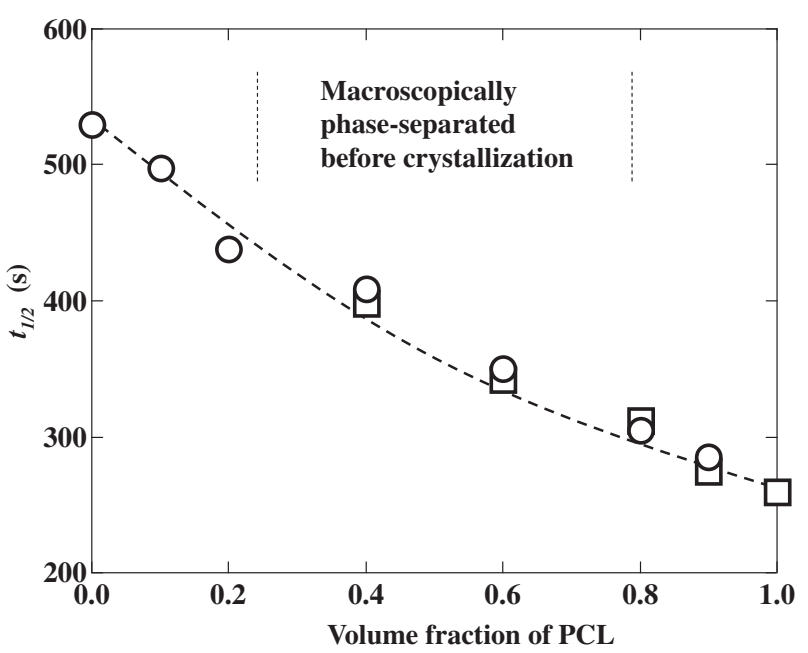

Figure 6. Half-time of crystallization $t_{1 / 2}$ at $40^{\circ} \mathrm{C}$ plotted against $\phi_{\mathrm{PCL}} \cdot t_{1 / 2}$ was evaluated by SAXS peaks from the PCL region $(\square)$ and PCL- $b$-PB region $(\bigcirc)$. The blends with $\phi_{\mathrm{PCL}}=0.4$ and 0.6 have liquid-liquid phase separation before crystallization.

can get the same result when the normalized peak intensity from the PCL region is plotted against $t$. The crystallization rate is faster with increasing $\phi_{\mathrm{PCL}}$, which is reasonable by considering crystallization rates of neat PCL and PCL- $b$-PB. The half-time of crystallization $\left(t_{1 / 2}\right)$ is a measure of the total rate of morphology formation and is plotted in Figure 6 against $\phi_{\mathrm{PCL}}$. In Figure $6, t_{1 / 2}$ for $\phi_{\mathrm{PCL}}=0.4$ and 0.6 is also included, where the phase separation occurs in advance by quenching and the crystallization starts from a macroscopically phase-separated structure, as easily expected from Figure 2. $t_{1 / 2}$ decreases continuously with increasing $\phi_{\mathrm{PCL}}$, and we can not detect any significant effect of the phase separation on $t_{1 / 2}$. A small domain size caused by the phase separation during quenching might be responsible, and we will investigate the size dependence of $t_{1 / 2}$ in the forthcoming manuscript. ${ }^{26}$

The normalized peak intensity is plotted against reduced time $\left(=t / t_{1 / 2}\right)$ in Figure 7 for various blends. Although the $t$ dependence of peak intensity shows different curves in Figure 5, they make one master curve in Figure 7, suggesting that the overall feature of morphology formation in our blend is equivalent to that of neat crystalline homopolymer (i.e., $\phi_{\mathrm{PCL}}=1$ ), where crystallization is the only factor to form the morphology. Therefore, the crystallization of PCL chains drives the overall rate of morphology formation in this blend, and the effect of phase separation does not appear in the viewpoint of crystallization rate. This conclusion does not mean that details of morphology formation are determined only by the crystallization of PCL chains, but by a combined effect of crystallization and phase separation, as we discuss in the next section. 
SR-SAXS Studies on Morphology Formation in a Binary Blend

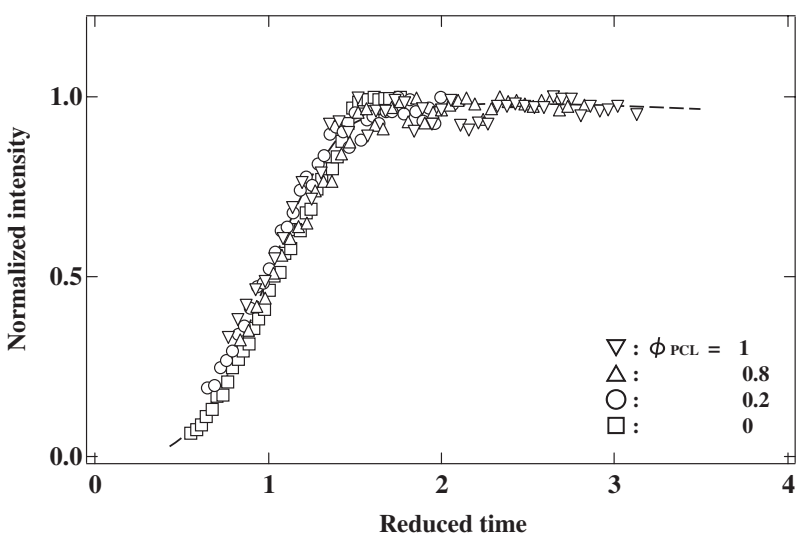

Figure 7. Normalized intensity plotted against reduced time $t / t_{1 / 2}$ for various blends crystallized at $40^{\circ} \mathrm{C}$.

\section{Volume Ratio of Two Crystallized Regions}

In our previous study, ${ }^{9}$ the volume ratio $(\theta(t))$ of two separated regions (crystallized and amorphous regions in Figure 1) at $t$ was evaluated by the combination of eq 1 and the SAXS intensity from crystallized regions, because we could not obtain any information about the growth rate of amorphous regions during morphology formation. In this study, on the other hand, the amorphous region is replaced with the PCL- $b$-PB region, so that the crystallization rate of this region is also available as well as that of the PCL region. Therefore, we can straightforwardly get the $t$ dependence of $\theta(t)$ from each SAXS peak.

We found in Figure $4 \mathrm{~b}$ that the crystallization rate of these two regions was identical, which imply that $\theta(t)$ is constant throughout the morphology formation if we assume that the volume of each region at $t$ is proportional to the normalized intensity. That is,

$$
\begin{aligned}
\theta(t) & \equiv \frac{V_{\mathrm{PCL}-b-\mathrm{PB}}(t)}{V_{\mathrm{PCL}}(t)+V_{\mathrm{PCL}-b-\mathrm{PB}}(t)} \\
\propto & \frac{\tilde{I}_{\mathrm{PCL}-b-\mathrm{PB}}(t)}{\tilde{I}_{\mathrm{PCL}-b-\mathrm{PB}}(t)+\frac{\phi_{\mathrm{PCL}}^{\prime}}{1-\phi_{\mathrm{PCL}}^{\prime}} \tilde{I}_{\mathrm{PCL}}(t)}
\end{aligned}
$$

where $\tilde{I}_{\mathrm{PCL}}(t)$ and $\tilde{I}_{\mathrm{PCL}-b-\mathrm{PB}}(t)$ are the normalized intensity from the crystallized PCL and PCL- $b$-PB regions at $t$, respectively, and $\phi_{\mathrm{PCL}}^{\prime}$ is the volume fraction of PCL in the blend. $\tilde{I}_{\mathrm{PCL}}(t)$ and $\tilde{I}_{\mathrm{PCL}-b-\mathrm{PB}}(t)$ are presented in Figure $4 b$, which tells us that they are equivalent irrespective of $t$, so that eq 4 reduces to $\theta(t)=1-$ $\phi_{\mathrm{PCL}}^{\prime} \approx 1-\phi_{\mathrm{PCL}}$. Therefore $\theta(t)$ is independent of $t$ and absolutely determined by the initial blend composition. This conclusion is the same with that previously obtained in a binary blend of PCL and PSO after numerical calculation. In this study, however, it comes simply from the experimental fact that the crystallization rate of two separated regions is identical (i.e.,

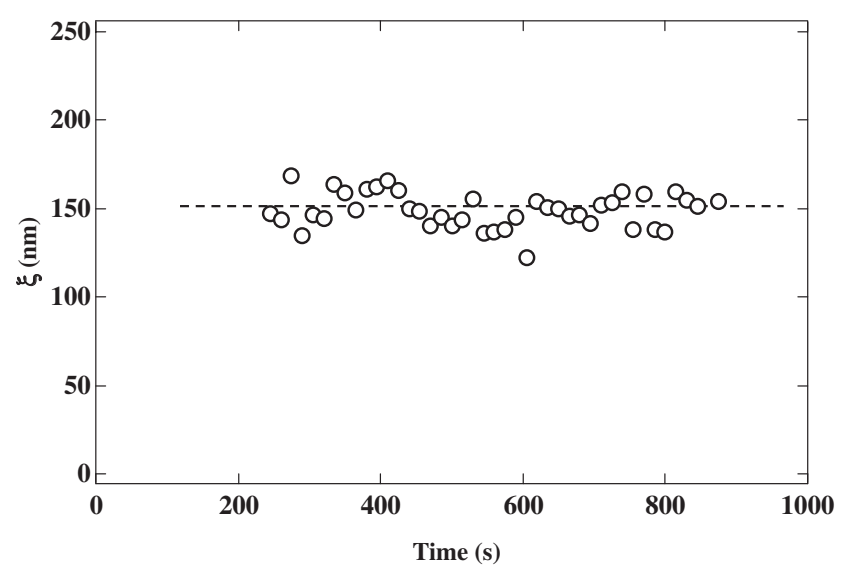

Figure 8. Size of heterogeneity in the two-phase structure, estimated from eq 1 , plotted against $t$ for the blend with $\phi_{\mathrm{PCL}}=$ 0.8 crystallized at $40^{\circ} \mathrm{C}$.

Figure 4b) irrespective of $t$, for which the (solidsolid) phase separation below Tm might participate in the morphology formation. However, we cannot definitely indicate that how this phase separation affects the morphology formation in our blend.

\section{Correlation Length of Two-Phase Structures}

We can evaluate correlation length $(\xi)$, size of heterogeneity in two-phase structures, by applying eq 1 to the increasing scattering toward $s \rightarrow 0$, and it is plotted in Figure 8 against $t$ for the blend with $\phi_{\mathrm{PCL}}=0.8 . \xi$ is almost constant throughout the morphology formation, which agrees with our previous conclusion. ${ }^{9}$ This fact gives additional information in the morphology formation of this blend. That is, $\xi$ is considered to be an average distance between the crystallized PCL and PCL- $b$-PB regions in the twophase structure, so that Figure 8 indicates that the average size of both regions keeps unchanged throughout the morphology formation.

The above conclusion, together with our previous conclusions that $\theta(t)$ is constant irrespective of $t$ and the volume of two-phase structures increases with $t$, confirms that the morphology in our blend is formed by a mechanism schematically illustrated in Figure 1, where substantial differences are that the amorphous region is replaced with the crystallized PCL- $b$-PB region and the matrix is a microphase-separated phase instead of an amorphous phase. When the blend is quenched from the microphase-separated melt, the crystallization of PCL chains starts after some induction time to yield two-phase structures consisting of crystallized PCL and PCL- $b$-PB regions. They increase in volume with $t$ and eventually prevail in the blend though the volume ratio of two regions is constant throughout the morphology formation. 


\section{CONCLUSIONS}

We have investigated the morphology formation in a binary blend of poly ( $\varepsilon$-caprolactone) homopolymer (PCL) and poly( $\varepsilon$-caprolactone)-block-polybutadiene copolymer (PCL- $b$-PB) by synchrotron small-angle $\mathrm{X}$-ray scattering (SR-SAXS). This blend has an UCST-type phase separation and the crystallization of PCL chains (i.e., PCL and PCL blocks in PCL- $b$ $\mathrm{PB})$ at a same temperature range, so that two factors may work simultaneously to yield a complicated morphology formation. The following results are obtained from this study.

1) Two regions, crystallized PCL and PCL- $b-\mathrm{PB}$ regions, appeared during the morphology formation when the blend was directly quenched from the microphase-separated melt. The crystallization rate of PCL was equal to that of PCL blocks in PCL- $b$-PB, and the overall rate of morphology formation was driven only by the crystallization of PCL chains.

2) The volume ratio of these two regions was constant throughout the morphology formation, which was straightforwardly obtained from the same crystallization rate of two regions. The solid-solid phase separation between crystallized PCL and PCL- $b$-PB participated in the details of morphology formation.

3) Even if the blend was quenched by passing through the UCST region for crystallization, the morphology formation was indistinguishable from the case without phase separation. The domain size formed by the liquid-liquid phase separation will be an important factor for the subsequent morphology formation, and the results will appear in the forthcoming manuscript. ${ }^{26}$

Acknowledgment. This study was partially supported by Grand-in-Aid for Scientific Research on Priority Areas, "Dynamic Control of Strongly Correlated Soft Materials" (No. 14045225). The SAXS measurement has been performed under the approval of the Photon Factory Program Advisory Committee (Nos. 2002G095 and 2004G093).

\section{REFERENCES}

1. D. R. Paul and S. Newman, "Polymer Blends," Vol. 1, Academic Press, New York, N.Y., 1978.

2. L. A. Utracki, "Polymer Alloys and Blends," Hanser Gardner Publications, Cincinnati, OH, 1989.

3. R. J. Roe and W. C. Zin, Macromolecules, 17, 189 (1984).

4. S. Nojima and R. J. Roe, Macromolecules, 20, 1866 (1987).

5. A. Adedeji, A. M. Jamieson, and S. D. Hudson, Polymer, 36, 2753 (1995).

6. H. K. Lee, C. K. Kang, and W. C. Zin, Polymer, 37, 287 (1996).

7. J. Bodycomb, D. Yamaguchi, and T. Hashimoto, Macromolecules, 33, 5187 (2000).

8. N. Y. Vaidya and C. D. Han, Polymer, 43, 3047 (2002).

9. S. Nojima, K. Kato, M. Ono, and T. Ashida, Macromolecules, 25, 1922 (1992).

10. H. L. Chen, J. C. Hwang, J. M. Yang, and R. C. Wang, Polymer, 39, 6983 (1998).

11. G. Matsuba, K. Shimizu, H. Wang, Z. Wang, and C. C. Han, Polymer, 44, 7459 (2003).

12. H. Tanaka and T. Nishi, Phys. Rev. Lett., 55, 1102 (1985).

13. S. Nojima, Y. Terashima, and T. Ashida, Polymer, 27, 1007 (1986).

14. R. M. Briber and F. Khoury, Polymer, 28, 38 (1987).

15. H. Tanaka and T. Nishi, Phys. Rev. A, 39, 783 (1989).

16. S. Nojima, K. Satoh, and T. Ashida, Macromolecules, 24, 942 (1991).

17. J. P. Penning and R. St. J. Manley, Macromolecules, 29, 84 (1996).

18. L. Z. Liu, B. Chu, J. P. Penning, and R. St. J. Manley, Macromolecules, 30, 4398 (1997).

19. J. C. Lee, H. Tazawa, T. Ikehara, and T. Nishi, Polym. J., 30, 780 (1998).

20. S. Hirano, Y. Nishikawa, Y. Terada, T. Ikehara, and T. Nishi, Polym. J., 34, 85 (2002).

21. S. Nojima, K. Kato, S. Yamamoto, and T. Ashida, Macromolecules, 25, 2237 (1992).

22. S. Nojima, Y. Akutsu, A. Washino, and S. Tanimoto, Polymer, 45, 7317 (2004).

23. G. Will, M. Bellotto, W. Parrish, and M. Hart, J. Appl. Crystallogr., 21, 182 (1988).

24. Y. Loo, R. A. Register, and A. J. Ryan, Phys. Rev. Lett., 84, 4120 (2000).

25. S. Nojima, M. Toei, S. Hara, S. Tanimoto, and S. Sasaki, Polymer, 43, 4087 (2002).

26. M. Akaba and S. Nojima, Polym. J., in press. 